\title{
The Consistency between Neutrophilic Alopecia in Clinical Form and Confirmation of Histology in Patients
}

\author{
Ehsani A, Nourmohammadpour P, Shojaei T and Beigi PK* \\ Misdiagnosis Association, USA
}

*Corresponding author: Dr. Pooya Khan Beigi, Misdiagnosis Association, 244 NW

Market Street Seattle, WA, 98107 USA, Tel: 1-206-299 0940, Email: misdiagnosis.association@gmail.com

\section{Research Article \\ Volume 3 Issue 1}

Received Date: November 11, 2017

Published Date: February 15, 2018

DOI: $10.23880 /$ cdoaj-16000141

\section{Abstract}

Alopecia has two types: Citacricial Alopecia and Non-Citacricial Alopecia. In Citacricial Alopecia, epithelium follicles are replaced by connective tissue which is irreversible. Non-Citacricial Alopecia is reversible and hair follicle remains intact. The Citarcricial and non-Citacricial share similarities, in both clinical and in histological aspects. Citacricial Alopecia illustrates a set of diseases; where the sign of which is destruction of skin biopsy. Few papers have investigated this topic. Citacricial Alopecia also has two types; the first type causes inflammation in initial and secondary follicles. While in second type doesn't harm the follicles. The first type further divides into neutrophilic, lymphocytic and mixed. This paper discusses the first type of Alopecia Scarring, which includes Acne Kolidal Splitting Cellulite and scalp dermatitis Pustular neutrophilic. In this research, all patients suspected of neutrophilic Alopecia from 2005-2009 were treated clinically and after that they went under skin biopsy examinations and histology study in order to confirm or reject the diagnosis; this had been performed to determine consistency between neutrophilic Alopecia in clinical form and confirmation of histology in these patients; The applied objective in this research deals with quick identification of neutrophilic Alopecia scarring and its quick treatment which could slow down the inflammation.

Keywords: Alopecia; Neutrophilic Alopecia; Decictiny Cellulitis; Folliculitis Declvance; Acne Kolidal

\section{Introduction}

\section{Research History and Method Analysis}

Acne Kolidal is a type of Scarring Alopecia, in terms of histology; similar to other types of initial Alopecia scarring. Severe subclinical disease may be observed in patients suffering from Acne Kolidal and it may be rooted from other cases.
Anti-inflammation drugs, which are used for other types of Alopecia, may be effective in treatment of this disease.

Decictiny Cellulitis is not a prevalent disease and it only covers $1-2 \%$ of the cases of Alopecia scarring. In this disease, infectious inflammation of sweat glands and round acnes are present. The disease with multiple indurate nodules is focused on vertex, crown and occipital 
area; these inflamed nodules develop very quickly and with no need to reach surface, it may lead in elliptical secretion and pain. This may last for years and at the end, fibrosis and hypertrophic scarring will be the remnant from the disease [1-5].

Acne Kolidal includes $11 \%$ of Alopecia scarring cases. This initial phase of the disease is apparent with small, smooth and firm papules. It is mostly seen in occipital areas. Acne Kolidal may be without pain, or with mild irritation and itching. The cause of the disease is unknown.

Main Goal of Project: Demographic investigation for the types of neutrophilic Alopecia was performed for patients underwent biopsy at Razi Hospital between the years 2005-2009.

\section{Material and Methods}

\section{Investigation Method}

Type of Study and its Implementation Method:

This case study covers all patients underwent biopsy at Razi Hospital between the years 2005-2009 and showed neutrophilic type of Alopecia scarring in pathology.

All required information existing in files were registered in special forms and then, patients information were analyzed.

\section{Sample Volume}

Among about 30,000 studied cases, 135 of which were diagnosed with the disease. All of the 135 cases were studied during the period.

Data Analysis Method: SPSS 11.5

\begin{tabular}{|c|c|c|}
\hline Variable & Independent & Dependent \\
\hline Age & + & \\
\hline Gender & + & \\
\hline Duration & + & \\
\hline Types & + & \\
\hline
\end{tabular}

Table 1: Table of Variables.

\section{Results}

Average age of the patients was 32 years old with standard deviation of 10.6 .
Average age of the patients suffering from Folliculitis Declvance was 31.89 years old.

Average age of the patients suffering from Decictiny Cellulitis was 32.4 years old.

Average age of the patients suffering from Acne Kolidal was 52 years old.

Average age of male patients suffering from Folliculitis Declvance was 30.97 years old and it was 33.4 years old for the female patients.

Age average of male patients suffering from Decictiny Cellulitis was 33.58 years old and for female patients was 28 years old.

The youngest patient suffering from Folliculitis Declvance was 16 years old and the oldest patient was 52 years old. The youngest for patients suffering from Decictiny Cellulitis was 18 years old and the oldest patient was 70 years old.

The youngest patients suffering from Acne Kolidal was 50 years old and the oldest was 54 years old.

\section{Results Related to Gender}

Among 135 identified cases, 97 were males and 38 were females; $71.9 \%$ were males and $28.1 \%$ were females.

In Folliculitis Declvance cases, $71 \%$ were males and 29\% were females.

In Decictiny Cellulitis cases, $68 \%$ were males and $32 \%$ were females.

In Acne Kolidal cases, $100 \%$ were males.

\begin{tabular}{|c|c|c|}
\hline & Frequency & Percentage $\%$ \\
\hline Valid Males & 97 & 71.9 \\
\hline Females & 38 & 28.1 \\
\hline Total & 135 & 100 \\
\hline
\end{tabular}

Table 2: Sexual Distribution of Patients in the Study.

\section{Results Related to Types of Alopecia}

Among 135 cases, most of the patients were involved with Folliculitis Declvance, with 98 cases $(72.6 \%)$. 35 cases related to Decictiny Cellulitis (25.9\%). Only 2 cases belonged to Acne Kolidal (about 1.5\%).

\begin{tabular}{|c|c|c|}
\hline & Frequency & Percentage \% \\
\hline Valid Acne Kolidal & 2 & 1.5 \\
\hline Decictiny Cellulitis & 35 & 25.9 \\
\hline Folliculitis Declvance & 98 & 72.6 \\
\hline Total & 135 & 100 \\
\hline
\end{tabular}

Table 3: Frequency of types of Alopecia. 


\section{Results Related to Duration}

Average duration between these 135 cases was 4.2 years with standard deviation of 3.9 years.

Average duration for females was 4.7 years and average duration for males was 4.03 years.

Average duration for Folliculitis Declvance was 4.7 years. Average duration for Decictiny Cellulitis was 1.9 years.

Average duration for Acne Kolidal for the two cases was 17.5 years.

Minimum duration was 3 months and maximum duration was 30 years.

Minimum duration for Folliculitis Declvance was 3 months and maximum duration was 20 years.

Minimum duration for Decictiny Cellulitis was 4 months and maximum duration was 12 years.

Minimum duration for Acne Kolidal was 5 years and maximum duration was 30 years.

\section{Discussion and Conclusion}

In this study among 135 cases, most of the patients had Folliculitis Declvance and while fewest had Acne Kolidal. In another study, Acne Kolidal covered $11 \%$ of the cases. This difference may be related to higher prevalence rate of Acne Kolidal among the black people in addition to the fact that clinical diagnosis of the disease is easier than others. As a result, there is usually no need for biopsy test to diagnose the disease. As this study was based on biopsy results, the difference in the results can be explained. Prevalence of Decictiny Cellulitis covers 1-2\% of Alopecia scarring patients and it also covers about $20-25 \%$ of Alopecia scarring in neutrophilic type and in our study, its prevalence was $25 \%$.

Our results indicated that $71.9 \%$ of cases were male and that the prevalence of neutrophilic Alopecia is higher in males. These data are supported by previous studies and articles.

In this study, 6 new cases were founded in a year for Folliculitis Declvance; of which, 1 case was female (17\%) and 5 cases were males (83\%). We studied 135 cases; of which, $71 \%$ of patients were males and $29 \%$ of patents were females. The difference could be explained by the fact that Iranian women tend to visit doctors for such cases less often than males.

In that study, minimum and maximum ages respectively were 17 and 62 years old; while in our study, minimum and maximum ages respectively were 16 and 54 years old. This shows no significant difference among the data of the two studies.

Average age of the patients was 36 years old; but in our study, it was almost 32 years old.

Duration average in that study was 3.2 years; but in our study, it was almost 3.7 years.

The maximum and minimum ages of patients with Decictiny Cellulitis respectively were between 18 and 40 years old. However, average age in our study was almost 32 years old. In our study, minimum and maximum age of the patients respectively was 17 and 70 years old.

Prevalence rate of Acne Kolidal among the blacks is higher; but, both cases founded in our study were in the same age (50 years old); this result may deal with the low number of biopsy cases.

\section{References}

1. Tan E, Martinka M, Ball N, Shapiro J (2004) Primary cicatricial alopecias: Clinicopathology of 112 cases. J Am Acad Dermatol 50(1): 25-32.

2. Sperling LC, Cowper SE (2006) The histopathology of primary cicatricial alopecia. Semin Cutan Med Surg 25(1): 41-50.

3. Somani N, Bergfeld WF (2008) Cicatricial alopecia: classification and histopathology. Dermatologic Therapy 21(4): 221-237.

4. Sperling LC, Homoky C, Pratt L, Sau P (2000) Acne Keloidalis is a form of Primary Scarring alopecia. Arch Dermatol 136(4): 479-484.

5. Van Exel CE, English JC $3^{\text {rd }}$ (2007) Erosive pustular dermatosis of the scalp and nonscalp. J Am Acad Dermatol 57(2): S11-14. 